\title{
Performance Comparison of Control Design for Unmanned Underwater Vehicle
}

\author{
Sung-Hyeon Joo*, Seon-Je Yang*, Tae-Yong Kuc®*, Jong-Koo Park", Yong-Serk Kim, \\ Nak-Yong $\mathrm{Ko}^{\text {*** }}$ and Yong-Seon Moon ${ }^{\text {*to }}$ \\ *Department of Electrical and Electronic Engineering, Sungkyunkwan University, Suwon, Korea \\ ***epartment of Electronic Engineering, Chosun University, Gwangju, Korea \\ *** Department of Electronic Engineering, Sunchon National University, Sunchon, Korea
}

\author{
무인 잠수정의 제어 성능 비교 연구 \\ 주성현 ${ }^{*}$ 양선제 ${ }^{*}$ 국태용이 ${ }^{*}$ 박종구 ${ }^{*}$ 김용석 ${ }^{*}$ 고낙용 ${ }^{* *}$ 문용선 ${ }^{* * *}$ \\ "성균관대학교 전자전기컴퓨터공학과 \\ *조선대학교 전자공학과 \\ 순천대학교 전자공학과
}

KEY WORDS: Backstepping control 백스텝핑 제어, Phase-locked-loop control 위상 고정 루프, Underwater vehicle 수중 운동체, Remotely operated vehicle 원격조종장비, Unmanned underwater vehicle 무인 잠수정

ABSTRACT: In this paper, we propose an adaptive backstepping controller to control the exact position and orientation of a remotely operated underwater vehicle with parametric model uncertainty. To further improve the angular velocity control precision of each thruster, a phase locked loop (PLL) controller has been added to the backstepping controller. A comparison of two backstepping controllers with and without the PLL control loop has been performed using simulations and experiments. The test results showed that the tracking performance could be improved by using the PLL control loop in the proposed adaptive backstepping controller.

\section{1. 서 론}

무인 잠수정(UUV, Unmanned underwater vehicle) 중 대표적인 $\mathrm{ROV}$ (Remotely operated vehicle)는 원격으로 조종되는 심해자원 탐사 및 개발용 무인 잠수정이다. 육상 또는 수상의 운용시스템 에서 원격으로 제어가 가능하여 사람이 작업하기 힘든 해저 환 경에서 사람을 대신해 해저 자원의 탐사, 침몰된 선박의 인양 작업, 바다 밑바닥의 기름 제거 작업, 해저 케이블 설치, 각종 수중 구조물의 설치 및 수리 등에 사용된다. 이러한 수중환경은 조류의 방향이나 속도가 깊이에 따라 달라지고 변화가 심하므 로 다양한 임무수행을 하는 ROV의 자세 유지를 위한 정밀한 제어시스템이 필요하다. 따라서 ROV와 같은 무인 잠수정의 활 용이 늘어나는 최근 추세에 맞추어 무인 잠수정의 제어를 위한 다양한 제어기법의 개발과 적용 연구가 보고되고 있다. 예를 들
면, 슬라이딩 모드 제어(Sliding mode control)의 경우 선형화 없 이 직접 비선형 동역학을 처리할 수 있는 장점을 가진 반면 제 어방법이 복잡하고 많은 변수를 처리하기 어려운 점이 있다 (Soylu et al., 2008; Raygosa-Barahona et al., 2011). 외부 환경의 변화에 따른 시스템 모델의 불확실성에 대처하기 위한 적응 제 어(Adaptive control)의 경우 모델 기반 적응제어는 이상적인 모 델을 만들어 추종 신호를 생성하는 방식과, 비모델 기반 적응제 어는 제어 성능 특성을 나타내는 평가값을 정해 이를 기반으로 제어 변수를 바꾸는 방식이 있다. 그러나 비선형 모델에 대해서 많은 연산량이 요구되고, 모델이 복잡할수록 적용하기가 어렵 다는 단점이 있다(Antonelli et al,. 2004).

또 다른 제어 기법 중 신경 네트워크 제어(Neural network control)는 제어대상과 관련된 변수들을 설정하고 이들의 결합과 결합의 가중치를 변수로 설정하는 제어 기법이다. 이 제어 기법

Received 21 August 2017, revised 1 February 2018, accepted 5 February 2018

Corresponding author Tae-Yong Kuc: +82-31-290-7137, tykuc@skku.edu ORCID: https://orcid.org/0000-0002-5816-0088

It is noted that this paper is revised edition based on proceedings of KMRTS 2017 in Pangyo.

(C) 2018, The Korean Society of Ocean Engineers

This is an open access article distributed under the terms of the creative commons attribution non-commercial license (http://creativecommons.org/licenses/by-nc/3.0) which permits unrestricted non-commercial use, distribution, and reproduction in any medium, provided the original work is properly cited. 
은 가중치를 결정하는 중간층 설계가 어렵다. 그리고 층의 개수 가 증가하면 제어 정밀도는 증가하지만 연산량이 증가하여 많 은 연산시간이 요구된다(Yuh, 1990).

그 외 비선형 추종제어 방법 중 하나인 백스텝핑 제어 (Backstepping control)는 목표치가 변할 때 그것을 제어량이 뒤 쫓아 가는 제어 방식이다. 이 기법의 단점은 급격한 출력의 변 화가 일어날 수 있다는 것이다. 하지만 다른 제어 기법을 함께 사용한다면 정밀한 제어가 가능하다(Wu and Karkoub., 2014.).

위에 제시된 대부분의 제어기법은 비선형 동역학을 모델링하 고, 다양한 환경변수들을 처리하는데 한계를 가진다. 또한 시스 템 동역학을 고려한 제어기법들의 경우 피드백 제어루프에 모 터 등 추진기의 특성을 반영하고 있지 않아 환경이 많이 변하 는 해양환경에서 작업 및 임무수행을 하는데 정확하고 빠른 대 응이 어려울 것으로 예상된다. 동적 작업 및 임무수행을 위해서 는 정확한 자세와 속도제어가 필요하고, 환경이나 시스템의 동 적 특성에 맞는 신속한 제어를 위하여 모터 제어루프를 고려할 필요가 있다.

본 논문에서는 임무를 수행하기 위해 ROV가 이동해야할 목 표점이 주어질 경우, 속도 프로파일을 통한 목표 속도 생성부터 모터 제어까지 통합된 제어 방법을 제시한다. 외부 교란과 유체 역학계수에 따른 적응 백스테핑 제어(Adaptive backstepping control)와 위상 고정 루프(PLL, Phased locked loop) 제어 방법을 제시하고 시뮬레이션 및 실험을 통해 PLL 모터제어 루프를 추 가함으로써 제어성능을 높일 수 있음을 검증하고자 한다. 2장에 서 ROV의 모델을 도입하고, 3장에서는 백스테핑과 PLL제어기 를 설계한다. 4장의 시뮬레이션과 5장의 실험을 통하여 위에서 소개한 PLL을 추가한 백스태핑 제어기와 독립적인 백스태핑 제 어기의 성능을 비교한다. 끝으로 6장에서 연구결과를 요약하고 결언을 제시한다.

\section{2. $\mathrm{ROV}$ 모델}

본 논문에서는 Fig. 1과 같이 Surge, Sway, Yaw 운동에 영향 을 주는 4개의 수평 트러스터와 Heave, Roll, Pitch 운동에 영향 을 주는 2 개의 수직 트러스터가 장착된 $\mathrm{ROV}$ 모델을 사용하였

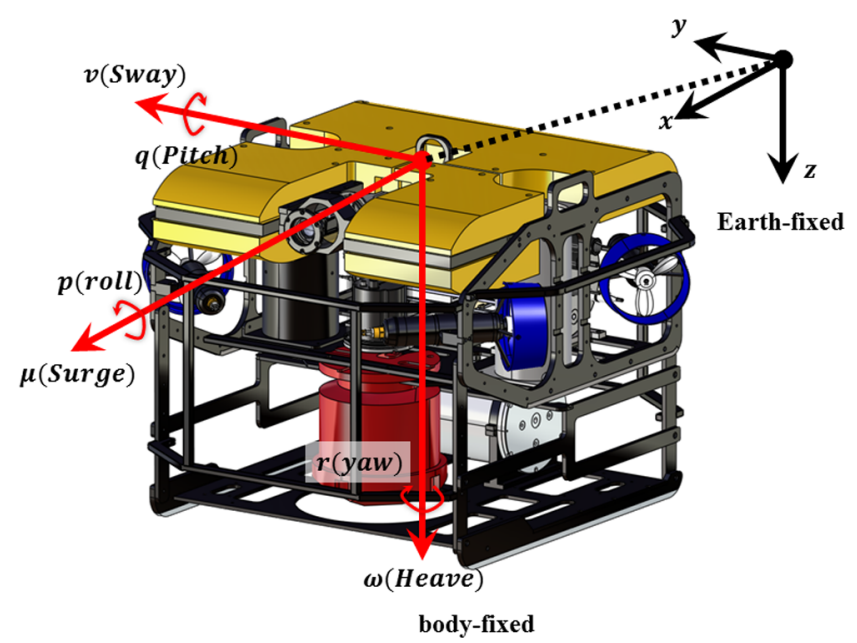

Fig. 1 Redone-technologies aROV100
다. 수직 트러스터의 장착 위치가 Roll, Pitch 운동에 많은 영향 을 주지 않기 때문에 Roll과 Pitch운동에 대해서는 고려하지 않 았다. 또한 ROV를 원격제어하기 위한 Tether cable의 영향은 무 시하기로 한다. 이 $\mathrm{ROV}$ 의 기구학 식은 다음 식 (1)과 같다.

$$
\begin{aligned}
& \dot{\eta}=J(\eta) \nu \\
& J(\eta)=\operatorname{diag}(R(\eta), T(\eta)) \\
& R(\eta)=\left[\begin{array}{ccc}
c_{\phi} c_{\theta}-s_{\phi} c_{\theta}+c_{\phi} s_{\theta} s_{\psi} & s_{\phi} s_{\theta}+c_{\phi} s_{\theta} c_{\psi} \\
s_{\phi} c_{\theta} & c_{\phi} c_{\psi}+s_{\phi} s_{\theta} s_{\psi} & -c_{\phi} s_{\psi}+s_{\phi} s_{\theta} c_{\psi} \\
-s_{\theta} & c_{\theta} s_{\psi} & c_{\theta} c_{\psi}
\end{array}\right] \\
& T(\eta)=\left[\begin{array}{ccc}
1 & s_{\psi} t_{\theta} & c_{\psi} t_{\theta} \\
0 & c_{\psi} & -s_{\psi} \\
0 & s_{\psi} / c_{\theta} & c_{\psi} / c_{\theta}
\end{array}\right]
\end{aligned}
$$

$\eta=[x, y, z, \phi, \theta, \psi]^{T}$ 는 전역좌표계(Earth-fixed frame)에서의 $x, y$, $z$ 의 위치와 Roll, Pitch, Yaw의 오일러각이고, $\nu=[\mu, v, w, p, q, r]^{T}$ 는 $\mathrm{ROV}$ 의 좌표계(Body-fixed frame)에서의 선속도와 각속도이다. 속 도 자코비안은 식 (2)와 같다. $\operatorname{Roll}(\phi)$ 에 대한 $\sin \phi$ 는 $s_{\phi}, \cos \phi$ 는 $c_{\phi}, \tan \phi$ 는 $t_{\phi}$ 로 나타내었고 $\operatorname{Pitch}(\theta), \operatorname{Yaw}(\psi)$ 에 대해서도 같은 방식 으로 나타내었다.

아래의 식 (3)은 ROV의 동역학이다.

$$
\dot{M}+C(\nu) \nu+D(\nu) \nu+G(\eta)+F=\tau
$$

$M \in R^{6 \times 6}$ 은 강체의 질량과 관성(Rigid body mass and inertia), 유체역학적 부가 질량(Hydrodynamic added mass)에 관한 행렬, $C \in R^{6 \times 6}$ 는 강체 코리올리와 구심성(Rigid body Coriolis and centripetal matrix)에 대한 행렬, $D \in R^{6 \times 6}$ 는 유체 저항에 대한 선 형 및 이차 감쇠에 대한 행렬, $G \in R^{6}$ 는 중력과 부력(Gravitation and buoyancy)에 대한 벡터 그리고 $F \in R^{6}$ 는 유체 운동에 의한 외 란(Disturbance)에 대한 항이다. $\tau \in R^{6}$ 는 $\mathrm{ROV}$ 트러스터의 힘과 모멘트에 대한 벡터이다(Lee et al., 2015; Fossen, 1994).

$\mathrm{ROV}$ 좌표계는 Fig. 1과 같이 무게중심(COM, Center of mass) 에 위치하고 있기 때문에 $M \in R^{6 \times 6}$ 과 $C \in R^{6 \times 6}$ 는 대칭 양의 정 부호행렬(Symmetric positive definite matrix)이고 반대칭행렬 (Skew-symmetric matrix)이다. $M \in R^{6 \times 6}$ 을 구성하고 있는 질량과 관성 행렬, 부가질량 행렬은 아래의 식 (4)와 같다.

$$
\begin{aligned}
& M=M_{r}+M_{h} \\
& M_{r}=\left[\begin{array}{cc}
m I_{3 \times 3} & O_{3 \times 3} \\
O_{3 \times 3} & {[I]_{x y z}}
\end{array}\right] \\
& {[I]_{x y z}=\left[\begin{array}{ccc}
I_{x x} & -I_{x y} & -I_{x z} \\
-I_{y z} & I_{y y} & -I_{y z} \\
-I_{z x} & -I_{z y} & I_{z z}
\end{array}\right]} \\
& M_{h}=-\left[\begin{array}{cc}
\operatorname{diag}\left(X_{\dot{\mu}}, Y_{\dot{v}}, Z_{\dot{\omega}}\right) & O_{3 \times 3} \\
O_{3 \times 3} & \operatorname{diag}\left(K_{\dot{p}}, L_{\dot{q}}, N_{\dot{r}}\right)
\end{array}\right]
\end{aligned}
$$

$\left\{X_{\dot{\mu}}, Y_{\dot{v}}, Z_{\dot{\omega}}, K_{\dot{p}}, L_{\dot{q}}, N_{\dot{r}}\right\}$ 는 각각 $x, y, z$ 방향에 따른 가속도 및 각 
가속도로 인한 힘과 모멘트의 유체역학적 계수이다. 이 값들은 힘과 모멘트를 해당하는 방향의 가속도와 각가속도의 편미분으 로 계산된다 $\left\{\frac{\partial X}{\partial \dot{\mu}}, \frac{\partial Y}{\partial \dot{v}}, \frac{\partial Z}{\partial \dot{\omega}}, \frac{\partial K}{\partial \dot{p}}, \frac{\partial L}{\partial \dot{q}}, \frac{\partial M}{\partial \dot{r}}\right\}$. 강체 코리올리와 구심 성에 대한 행렬은 아래의 식 (5)와 같다.

$$
C(\nu)=\left[\begin{array}{cc}
O_{3 \times 3} & C_{12} \\
C_{21} & C_{22}
\end{array}\right]
$$

여기에서 $C_{21}=-C_{12}^{T}, C_{22}=-C_{22}^{T}$ 이다.

$$
\begin{aligned}
& C_{12}=\left[\begin{array}{l}
m\left(y_{g} q+z_{g} r\right) \\
-m\left(x_{g} q-w\right)-Z_{\omega} \omega \\
-m\left(x_{g} r+\omega\right)-Y_{v} v
\end{array}\right. \\
& \left.\begin{array}{cc}
-m\left(y_{g} p+\omega\right)+Z_{\omega} \omega & -m\left(z_{g} p-v\right)-Y_{v} v \\
m\left(z_{g} r+x_{g} p\right) & -m\left(z_{g} r+\mu\right)+X_{\mu} \mu \\
-m\left(y_{g} r-\mu\right)+X_{\mu} \mu & m\left(x_{g} p+y_{g} q\right.
\end{array}\right] \\
& C_{22}=\left[\begin{array}{l}
0 \\
-I_{y z} q+I_{x z} p-I_{z z} r+N_{r} r \\
I_{y z} r+I_{x y} p-I_{y y} q+L_{q} q
\end{array}\right. \\
& \left.\begin{array}{cc}
I_{y z} q-I_{x z} p+I_{z z} r-N_{r} r & -I_{y z} r-I_{x y} p+I_{y y} q-L_{q} q \\
0 & -I_{x z} r-I_{x y} q+I_{x x} p-K_{p} p \\
I_{x z} r+I_{x y} q-I_{x x} p+K_{p} p & 0
\end{array}\right]
\end{aligned}
$$

유체 저항에 대한 선형 및 이차 감쇠 행렬은 아래의 식 (6)과 같다.

$$
\begin{aligned}
& D=\operatorname{diag}\left(D_{\mu}, D_{v}, D_{\omega}, D_{p}, D_{q}, D_{r}\right) \\
& D_{\mu}=-\left(X_{\mu}+X_{\mu \mu}|\mu|\right) \\
& D_{v}=-\left(Y_{v}+Y_{v v}|v|\right) \\
& D_{\omega}=-\left(Z_{\omega}+Z_{\omega \omega}|\omega|\right) \\
& D_{p}=-\left(K_{p}+K_{p p}|p|\right) \\
& D_{q}=-\left(L_{q}+L_{q q} \mid q\right) \\
& D_{r}=-\left(N_{r}+N_{r r}|r|\right)
\end{aligned}
$$

수중 로봇은 저밀도 유체에서 움직이는 동안 유체운동은 동 역학에 영향을 주어 운동에 방해하는 힘과 모멘트를 발생시킨 다. 이런 외란에 대한 힘과 모멘트 벡터는 아래의 식 (7)과 같이 정의하여 시스템 모델에 포함시킨다.

$$
F=\left[\begin{array}{c}
m_{f} \dot{v}_{l} \\
m_{f}\left(R_{b} \times \dot{v}_{l}\right)
\end{array}\right]
$$

여기에서 $m_{f}$ 는 로봇에 의해 이동된 유체의 질량이고 $v_{l}=$ $\left[\mu_{f}, v_{f}, \omega_{f}\right]^{T}$ 는 유체의 속도벡터, $R_{b}=\left[x_{b}, y_{b}, z_{b}\right]^{T}$ 는 부력 중심의 위치벡터 이다.

중력과 부력에 대한 벡터는 식 (8)과 같다.

$$
\begin{aligned}
& G(\eta)=\left[\begin{array}{l}
G_{f}(\eta) \\
G_{m}(\eta)
\end{array}\right] \\
& G_{f}(\eta)=\left[\begin{array}{c}
\left(W_{g}-W_{b}\right) s_{\theta} \\
-\left(W_{g}-W_{b}\right) c_{\theta} s_{\phi} \\
-\left(W_{g}-W_{b}\right) c_{\theta} c_{\phi}
\end{array}\right] \\
& G_{m}(\eta) \\
& =\left[\begin{array}{c}
-\left(y_{g} W_{g}-y_{b} W_{b}\right) c_{\theta} c_{\phi}+\left(z_{g} W_{g}-z_{b} W_{b}\right) c_{\theta} s_{\phi} \\
\left.-\left(z_{g} W_{g}-x_{b} W_{b}\right) c_{\theta} s_{\phi}-\left(x_{g} W_{g}-x_{b} W_{b}\right) c_{\theta} c_{b}\right) s_{\theta}
\end{array}\right]
\end{aligned}
$$

$\mathrm{ROV}$ 의 힘/모멘트 기구학은 아래의 식 (9)과 같이 주어진다.

$$
\tau=B u
$$

$u=\left[u_{1}, u_{2}, u_{3}, u_{4}, u_{5}, u_{6}\right]^{T}$ 는 트러스터의 출력을 나타내는 벡터이 다. 트러스터 구성 행렬(TCM, Thruster configuration matrix)은 $\mathrm{ROV}$ 의 6 개 트러스터의 기하학적 구조에 따라 결정된다. 여기 에서 $u_{1}$ 과 $u_{4}$ 는 수직 트러스터의 추력이며 $u_{2}, u_{3}, u_{5}, u_{6}$ 은 수 평 트러스터의 추력이다. TCM은 아래 식 (10)과 같다.

$$
B=\left[\begin{array}{llllll}
B_{x 1} & B_{x 2} & B_{x 3} & B_{x 4} & B_{x 5} & B_{x 6} \\
B_{y 1} & B_{y 2} & B_{y 3} & B_{y 4} & B_{y 5} & B_{y 6} \\
B_{z 1} & B_{z 2} & B_{z 3} & B_{z 4} & B_{z 5} & B_{z 6} \\
B_{\phi 1} & B_{\phi 2} & B_{\phi 3} & B_{\phi 4} & B_{\phi 5} & B_{\phi 6} \\
B_{\theta 1} & B_{\theta 2} & B_{\theta 3} & B_{\theta 4} & B_{\theta 5} & B_{\theta 6} \\
B_{\psi 1} & B_{\psi 2} & B_{\psi 3} & B_{\psi 4} & B_{\psi 5} & B_{\psi 6}
\end{array}\right]
$$

여기에서

$$
\begin{aligned}
& B_{x 2}=-B_{x 3}=B_{x 5}=-B_{x 6}=\cos \delta \\
& B_{y 2}=B_{y 3}=-B_{y 5}=-B_{y 6}=\sin \delta \\
& B_{z 1}=B_{z 4}=1 \\
& B_{\psi 2}=-B_{\psi 3}=-B_{\psi 5}=B_{\psi 6}=r \sin \beta \\
& B_{x 1}=B_{x 4}=0 \\
& B_{y 1}=B_{y 4}=0 \\
& B_{z 2}=B_{z 3}=B_{z 5}=B_{z 6}=0 \\
& B_{\phi 1}=B_{\phi 2}=B_{\phi 3}=B_{\phi 4}=B_{\phi 5}=B_{\phi 6}=0 \\
& B_{\theta 1}=B_{\theta 2}=B_{\theta 3}=B_{\theta 4}=B_{\theta 5}=B_{\theta 6}=0 \\
& B_{\psi 1}=B_{\psi 4}=0
\end{aligned}
$$

$\delta$ 는 $\mathrm{ROV}$ 좌표계에서 $x-y$ 평면상에서의 $x$ 축과 트러스터가 이루는 각도이고, $r$ 은 ROV의 무게중심과 트러스터까지의 거리 이다. $\beta=\delta+\alpha$ 이고, $\alpha$ 는 $\mathrm{ROV}$ 좌표계에서 $x-y$ 면상의 $x$ 축과 $r$ 벡터가 이루는 각도이다. 앞서 언급한 바와 같이 수직 트러스터 는 Heave, Roll, Pitch운동에 영향을 주지만 트러스터 장착 위치 로 인해 Roll과 Pitch운동에 대한 영향력은 무시하기로 하였으므 로 $B_{\phi i}(i=1,2,3,4,5,6)$ 와 $B_{\theta i}(i=1,2,3,4,5,6)$ 는 0 으로 두었다. 


\section{3. 제어기 설계}

\subsection{Backstepping control design}

이 논문에서 제시하고자 하는 백스테핑 제어 모델은 아래의 Fig. 2와 같이 구성된다.

$\nu^{*}=J^{-1}\left(\eta_{2}\right)\left(\dot{\eta_{d}}+\Lambda_{1} e_{\eta}\right)$

$\tau^{*}=Y(\dot{\nu}, \nu, \eta, \dot{\Omega}, \Omega) \hat{\Phi}+\Lambda_{2} e_{\nu}+J^{T}\left(\eta_{2}\right) e_{\eta}$

$Y(\dot{\nu}, \nu, \eta, \dot{\Omega}, \Omega)=\left[\begin{array}{lll}Y_{1} & \vdots & Y_{2}\end{array}\right]$

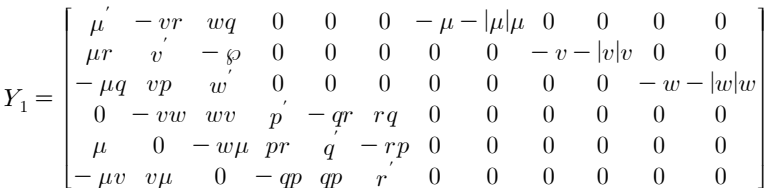

$Y_{2}=\left[\begin{array}{cccccccccccccc}0 & 0 & 0 & 0 & 0 & 0 & s \theta & 0 & 0 & 0 & \mu_{f}^{\prime} & 0 & 0 & 0 \\ 0 & 0 & 0 & 0 & 0 & 0 & -c \theta s \phi & 0 & 0 & 0 & v_{f}^{\prime} & 0 & 0 & 0 \\ 0 & 0 & 0 & 0 & 0 & 0 & -c \theta s \phi & 0 & 0 & 0 & w_{f}^{\prime} & 0 & 0 & 0 \\ -p-|p| p & 0 & 0 & 0 & 0 & 0 & 0 & c \theta c \phi-c \theta s \phi & 0 & \mu_{f}^{\prime} & 0 & 0 \\ 0 & 0 & -q-|q| q & 0 & 0 & 0 & -c \theta c \phi & 0 & -s \theta & 0 & 0 & v_{f}^{\prime} & 0 \\ 0 & 0 & 0 & 0 & -r-|r| r & 0 & c \theta s \phi & s \theta & 0 & 0 & 0 & 0 & w_{f}^{\prime}\end{array}\right]$

$\hat{\Phi}=\operatorname{Pr}[\hat{\Phi}(t-T)]+\frac{1}{\beta} Y^{T}\left(v^{\prime}, v, \eta, \Omega^{\prime}, \Omega\right) e_{v}$

$u=B^{\#} \tau^{*}$

$u_{i}=\rho_{i} \Omega_{i}^{2}$

식 (11)은 ROV의 목표속도 $\left(\nu^{*}\right)$ 를 구하는 식이다. 식 (1)과 식 (2)를 기반으로 위치의 오차를 보상해준다. $\eta_{2}=[\phi, \theta, \psi]^{T}$ 이고, $e_{\eta}=\eta_{d}-\eta$ 는 현재 위치 및 자세 $(\eta)$ 와 목표 위치 및 자세 $\left(\eta_{d}\right)$ 사 이의 에러값을 나타낸다. $\Lambda_{1}=\operatorname{diag}\left(\Lambda_{x}, \Lambda_{y}, \Lambda_{z}, \Lambda_{\phi}, \Lambda_{\theta}, \Lambda_{\psi}\right)$ 는 양의 이득값이다.

식 (12)는 ROV의 목표 힘과 모멘트를 구하는 식이다. Regression $\operatorname{matrix}(Y(\dot{\nu}, \nu, \eta, \dot{\Omega}, \Omega))$ 은 식 (3)을 기반으로 도출한 행렬로 트러스 터의 홀센서와 AHRS(Attitude and heading reference system)로 각 원소의 값을 계산하였다. Unknown parameter vector $\left(\Phi_{26 \times 1}\right)$ 는 $\mathrm{ROV}$ 의 불확실한 회전 모멘트와 유체역학 계수로 이루어진 벡터 이고, $\widehat{\Phi_{26 \times 1}}$ 는 식 (13)에 의해 계산된 Unknown parameter vector의 추정값이다. $\beta$ 는 1 보다 큰 양의 이득값이다. $e_{\nu}=\nu_{d}-\nu$ 는 $\mathrm{ROV}$ 좌 표계에서 목표 속도 $\left(\nu_{d}\right)$ 와 현재 속도 $(\nu)$ 사이의 에러값을 나타낸 다. $\Lambda_{2}=\operatorname{diag}\left(\Lambda_{x}, \Lambda_{y}, \Lambda_{z}, \Lambda_{\phi}, \Lambda_{\theta}, \Lambda_{\psi}\right)$ 는 양의 이득값이다.

식 (14)는 Inverse TCM에서 얻을 수 있는 식으로 식 (12)에서 계산된 힘과 모멘트 $\left(\tau^{*}\right)$ 를 4 개의 수평 트러스터와 2 개의 수직 트러스터의 추력으로 분리하기 위한 식이다. 이는 ROV의 기하 학적 구조에 따라 달라지며 식 (9)와 식 (10)에서 $\mathrm{TCM}$ 을 구하 였다. $B^{\#}$ 은 ROV의 힘과 모멘트를 수직 및 수평방향 트러스터 의 추력으로 분리해내기 위한 Pseudo inverse이다.

식 (15)는 Inverse Thruster Model이다. 이 식은 사용하는 트러 스터의 모델에 따라 결정된다. 이 논문에서 사용된 트러스터의 추력에 따른 트러스터의 회전속도에 대한 자료는 트러스터의 제조사인 레드원테크놀러지 사에서 제공받아 정리하였다. $i$ 는 트러스터의 번호이며 $\rho_{i}$ 는 비례상수, $\Omega_{i}$ 는 트러스터의 회전속도 이다.

\subsection{PLL control design}

PLL(Phased locked loop) 제어는 모터의 속도를 정밀하게 제어 하기 위해 설계하였다. 이 논문에서 제시하고자 하는 PLL 모터

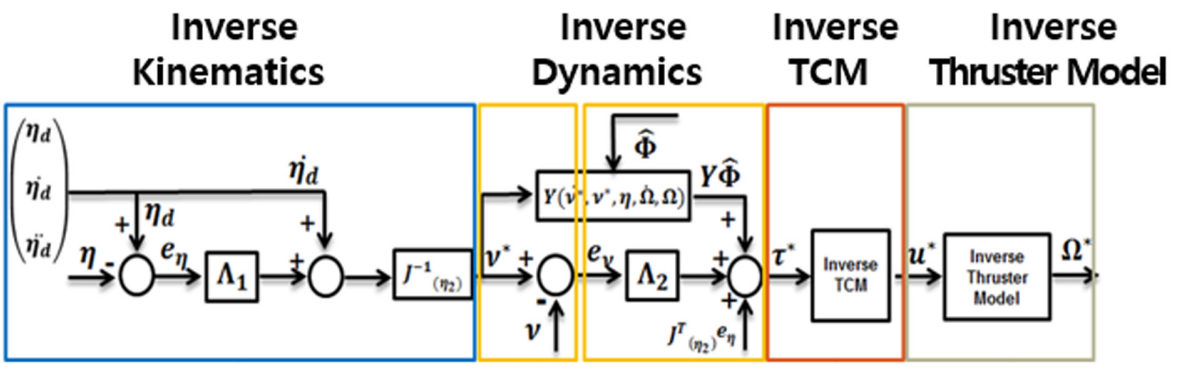

Fig. 2 Backstepping control block diagram

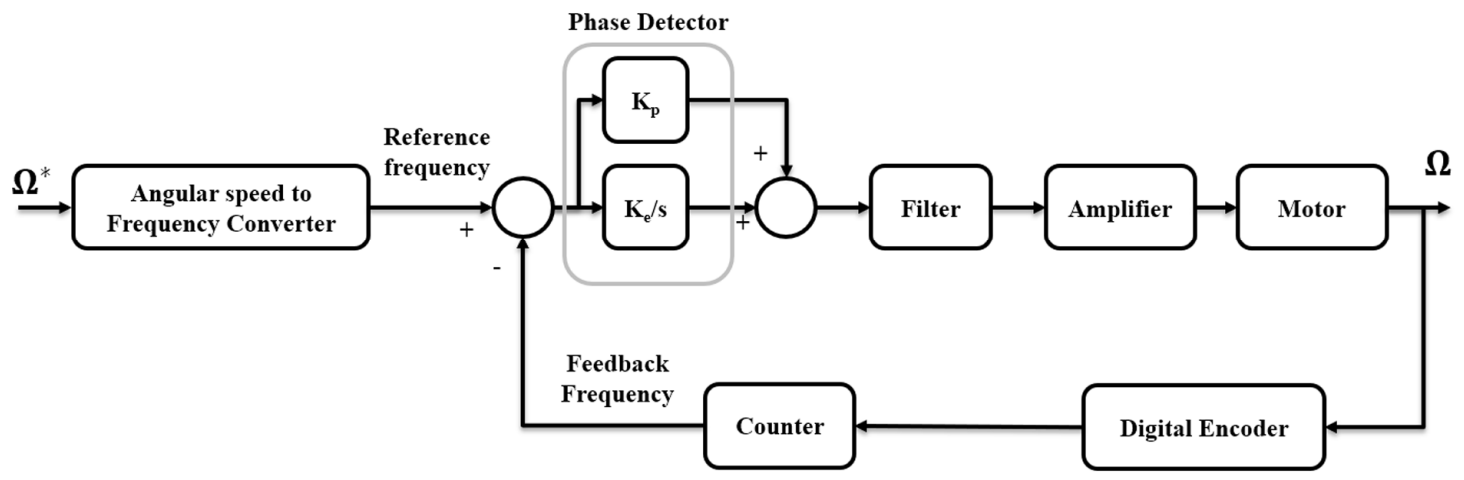

Fig. 3 Motor control block diagram using PLL controller 
제어 모델은 아래의 Fig. 3과 같다.

제안된 PLL 모터 제어 모델의 동작 원리는 다음과 같다. 변환 기를 사용하여 Backstepping 제어기에서 나온 출력을 주파수로 변화시켜 모터의 기준 주파수를 구한다. 그리고 모터의 회전속 도를 디지털 엔코더를 이용하여 측정 후 출력 주파수로 변환한 다. 이후 출력 주파수와 기준 주파수의 비교를 위해 카운터 (Counter)로 고정주파수와 출력주파수의 비율에 따라 나누는 방 식을 사용한다(Geiger, 1981; Wise., 1985). 출력 주파수와 기준 주파수의 차이를 위상 검출기(Phase Detector)에서 계산하고, PI 제어기를 이용하여 주파수를 보상해주는 입력 값을 생성한다. 필터에서는 위상 검출기(Phase Detector)로부터 나온 주파수 차 이에 대해 고주파 성분을 제거하고, 리플현상을 제거해 준다. 마지막으로 증폭기를 이용하여 모터의 최종 입력값을 계산한다.

\section{4. 시뮬레이션}

시뮬레이션은 National Instrument 사의 LabVIEW 2015버전을 사용하였고 원점을 중심으로 가로 $5 \mathrm{~m}$, 세로 $5 \mathrm{~m}$ 의 정사각형 경 로를 움직이면서 ROV의 위치와 경로에 대한 오차를 확인하였 다. 아래의 Fig. 4 는 경로를 움직일 때의 ROV의 $x, y$ 좌표를 나 타낸 그래프이다. 검은색 점선은 입력 경로, 파란색 선은 백스 테핑 제어기와 PLL제어기를 함께 사용하였을 때의 ROV의 자 취, 빨간색 선은 백스테핑 제어기만 사용하였을 경우 ROV의 자취를 나타낸 것이다. 그래프에서 보듯이 백스테핑 제어기만 사용하였을 때 보다 백스테핑 제어기와 PLL 제어기를 함께 사 용하였을 때 좀 더 입력 경로를 잘 따라 가는 것을 확인 할 수 있다.

\section{Backstepping controller + PLL vs. Backstepping controller}

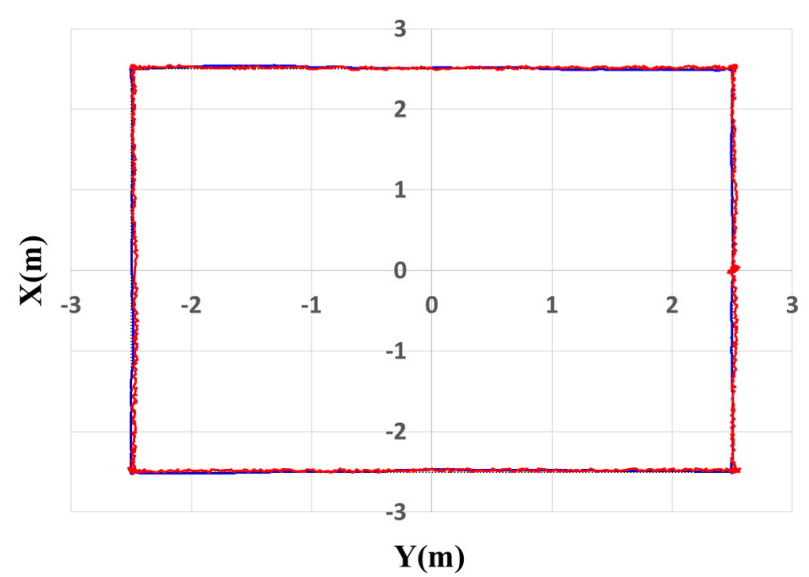

Fig. 4 Result of ROV execution simulation

Fig. 5와 Fig. 6은 입력 경로를 주행하는 동안 시간에 따른 $x$, $y$ 좌표를 나타낸 것이다. 여기에서도 백스테핑 제어기만을 사용 하였을 때보다 백스테핑 제어기와 PLL 제어기를 함께 사용할 경우 리플이 줄어들고 안정적인 경로 추종이 가능함을 볼 수 있다. 아래의 Table 1은 경로 주행 시 입력 $x, y$ 좌표와 출력 $x$, $y$ 좌표 상의 최대 오차값을 나타낸 것이다.

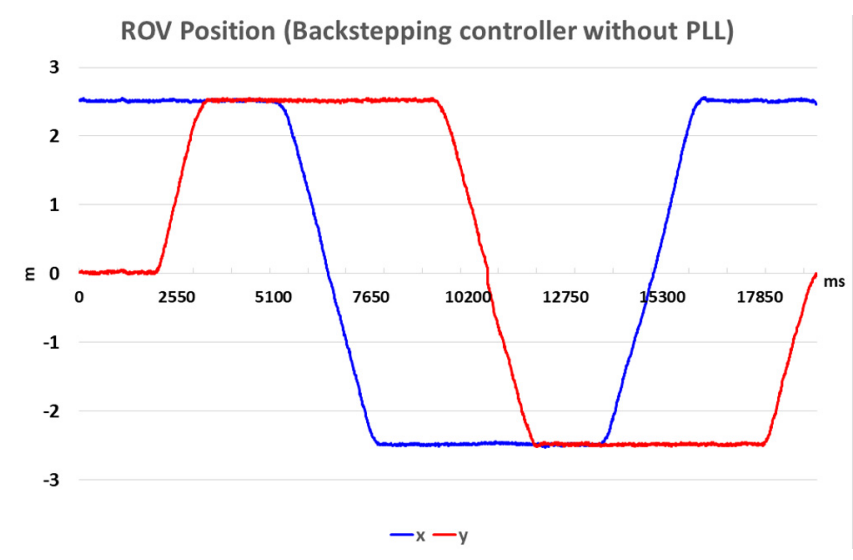

Fig. 5 Coordinate of $x$ and $y$ using backstepping controller only

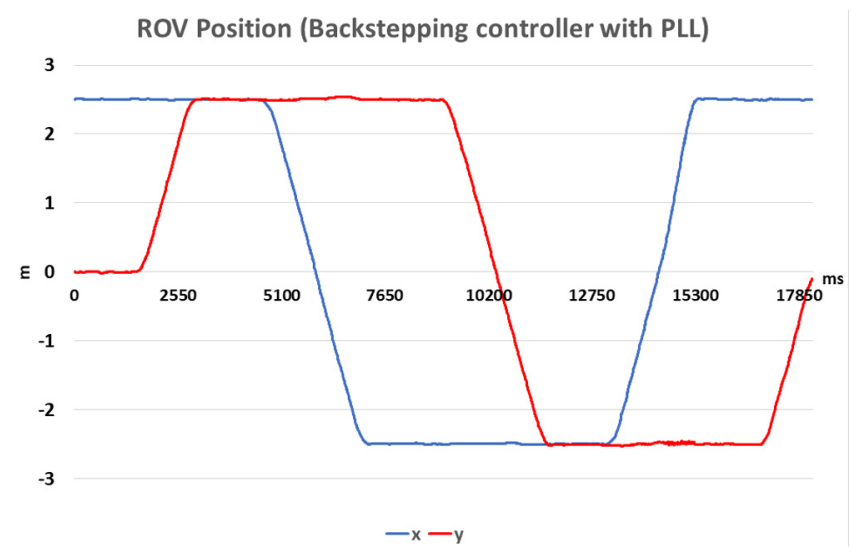

Fig. 6 Coordinate of $x$ and $y$ using backstepping and PLL controller

Table 1 ROV position maximum error according to input path

\begin{tabular}{ccc}
\hline \hline Error $[\mathrm{m}]$ & Backstepping + PLL & Backstepping \\
\hline$x$ Maximum & 0.0156 & 0.0563 \\
$x$ Maximum & 0.0181 & 0.0756 \\
\hline
\end{tabular}

\section{5. 실 험}

실험에 사용된 ROV는 Redone-technologies사의 aROV100이다. 이 ROV는 $200 \mathrm{~m}$ 정도에서 사용되는 ROV로 최대 속도 $1.3 \mathrm{~m} / \mathrm{s}$ (Surge 방향), $1.03 \mathrm{~m} / \mathrm{s}$ (Heave 방향)를 낼 수 있다. 무게는 $60 \mathrm{~kg}$ 정도 이며 전진 추력은 $24 \mathrm{kgf}$ 이다. 실험은 Redone-technologies사의 수중로 봇실험 수조에서 이루어 졌으며, 수조의 크기는 $5 \mathrm{~m} \times 5 \mathrm{~m} \times 5 \mathrm{~m}$ 이다.

Fig. 7(a)는 백스테핑 제어만을 통해 -Sway방향으로 이동시킨 결과이다. 흰색 점선은 처음 위치에서 ROV의 -Sway방향의 경 로이며, 흰색 점들은 이동하면서 측정한 ROV 중심의 위치이다. 실험 결과에서 보면 -sway방향으로 이동하면서 처음 경로에서 점차 벗어나고 있는 모습을 볼 수 있다. Fig. 7(b)는 백스테핑 제 어기와 PLL제어기를 함께 사용하여 Sway방향으로 이동시켰을 때의 결과이다. Fig. 7(a)와 마찬가지로 흰색 점선은 처음 위치 에서 ROV의 -Sway방향의 경로이며, 흰색 점들은 측정된 ROV 중심의 위치를 표현한 것이다. 백스테핑 제어만 사용하였을때 와는 달리 ROV의 중심이 처음 경로를 벗어나지 않고 따라가고 있는 모습을 볼 수 있다. 


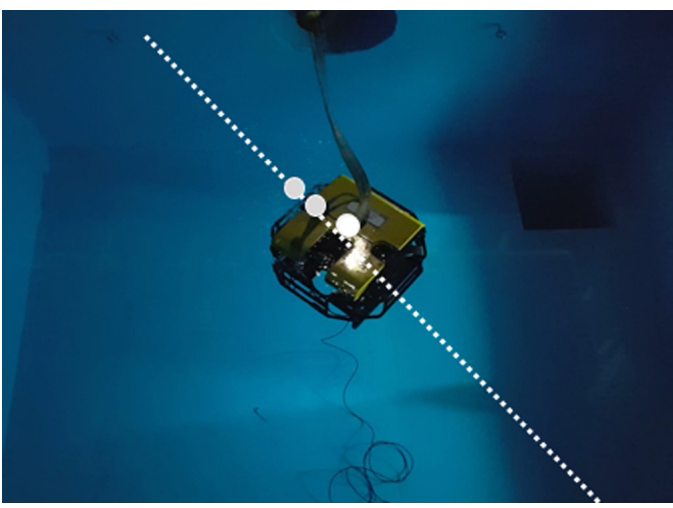

(a)

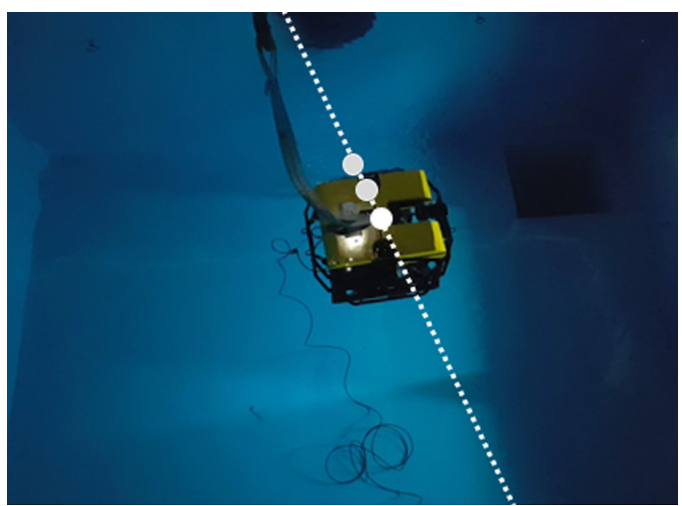

(b)

Fig. 7 (a) Sway direction path using backstepping controller only (b) Sway direction path using backstepping controller and PLL controller

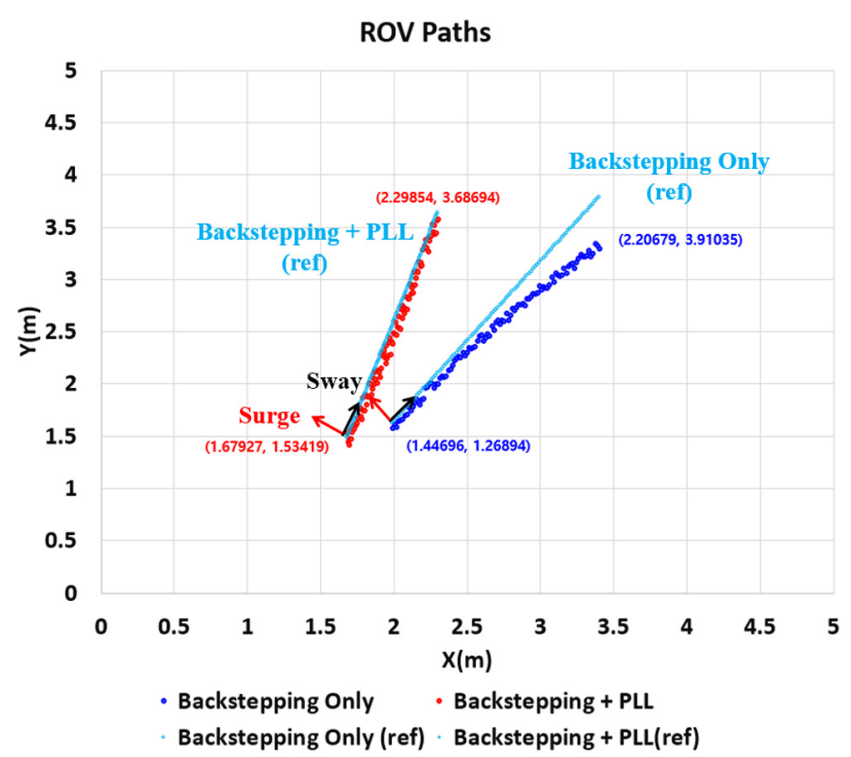

Fig. 8 ROV paths using backstepping controller with and without PLL controller

Table 2 ROV position maximum error according to input path

\begin{tabular}{ccc}
\hline \hline Error $[\mathrm{m}]$ & Backstepping + PLL & Backstepping \\
\hline Maximum & 0.0626 & 0.4895 \\
\hline
\end{tabular}

Fig 8 는 실험에서 측정된 $\mathrm{ROV}$ 의 이동경로를 수치화해서 표 현한 그래프이다. 빨간색 점들은 백스테핑 제어와 PLL 제어기 를 동시에 사용하였을 때 ROV가 -Sway방향으로 움직이면서 매 순간 측정한 ROV의 위치이고 파란색 점들은 백스테핑 제어만 을 이용했을 때의 ROV의 위치이다. 하늘색 선분은 제어기의 성 능을 평가하기 위하여 입력한 Reference 경로를 의미한다. Table 2 에서 알 수 있듯이 백스테핑 제어기와 PLL 제어기를 동시에 사용한 경우가 PLL 제어기를 사용하지 않은 경우보다 최대 오 차율이 적은 것을 알 수 있다.

\section{6. 결 론}

본 논문에서는 6 자유도 운동을 하는 $\mathrm{ROV}$ 의 위치 및 자세를
제어하기 위해 적응 백스테핑과 PLL제어 방법을 제시하였다. 컴퓨터 시뮬레이션을 통해 PLL제어를 사용하지 않았을 때와 비 교하여 적응 백스테핑과 PLL제어를 함께 사용하였을 때 위치 및 자세 제어가 안정적임을 보였다. 또한 Redone-technologies사 의 aROV100에 직접 적용함으로써 적응 백스테핑제어와 PLL제 어를 함께 사용한 경우가 ROV의 위치 및 자세제어에 더 안정 적임을 실험을 통하여 검증하였다.

\section{후 기}

이 논문은 산업통상자원부의 재원으로 엔지니어링 개발연구 센터(과제번호 : N0000990) 및 한국산업기술평가관리원(과제번 호 : 10063413)의 지원을 받아 수행된 연구이다.

\section{References}

Antonelli, G., Caccavalee, F., Chiaverini, S., 2004. Adaptive Tracking Control of Underwater Vehicle-Manipulator Systems Based on the Virtual Decomposition Approach. IEEE Transactions on Robotics and Automotion, 20(3), 594-602.

Geiger, D.F., 1981. Phaselock Loops for DC Motor Speed Control. Hoboken, NJ, USA.

Lee, H.J, Yang. S.J,, Park. J.G., Kuc. T.Y., Ko, N.Y., Moon, Y.S., 2015. PLL Controller for Precise Velocity Tracking of UUV: A new ROV Model and Its Control. Control, Automation and Systems (ICCAS), 15th International Conference, IEEE, 488-492.

Raygosa-Barahona, R., Parra-Vega. V., Olguin-Diaz. E., Munoz-Ubando. L., 2011. A Model-free Backstepping with Integral Sliding Mode Control for Underactuated ROVs. Electrical Engineering Computing Science and Automatic Control (CCE), 8th International Conference, IEEE.

Soylu, S., Buckham, B.J., Podhorodeski, R.P., 2008. A Chatteringfree Sliding Mode Control for Underwater Vehicles with Faulttolerant Infinity-norm Thrust Allocation. Ocean Engineering, 35(16), 1647-1659.

Fossen, T.I., 1994. Guidance and Control of Ocean Vehicles. Wiley, London, UK. 
Wise, M.G., 1985. Digital Phase-locked Loop Speed Control for a Brushless D.C. Motor. Naval Postgraduate School Monterey, CA.

Wu, H.M, Karkoub, M., 2014. Hierarchical Backstepping Control for Trajectory-tracking of Autonomous Underwater Vehicles
Subject to Uncertainties. Control, Automation and Systems (ICCAS), 14th International Conference, IEEE, 1191-1196. Yuh, J., 1990. A Neural Net Controller for Underwater Robotic Vehicles. IEEE Journal of Oceanic Engineering, 15(3), 161-166. 